\title{
Corrigendum
}

\section{Use of a best practice alert linking Clostridioides difficile infection test results to a severity-based treatment order set - CORRIGENDUM}

The title of the article by Reed, et al $^{1}$ was incorrect in the final published version. The original title should have read "Clostridioides." This has been updated in the original published article. The authors apologize for this error.

\section{Reference}

1. Reed, HL, Van Schooneveld, TC, Reha, CG, Bergman, SJ. Use of a best practice alert linking Clostridioides difficile infection test results to a severity-based treatment order set. Infection Control Hosp Epidemiol 2018;1-2. 